\title{
UNCONTROLLABILITY FOR LINEAR AUTONOMOUS MULTI-INPUT DYNAMICAL SYSTEMS DEPENDING ON PARAMETERS*
}

\author{
ALEXEI A. MAILYBAEV ${ }^{\dagger}$
}

\begin{abstract}
Linear multi-input dynamical systems smoothly depending on parameters are considered. A set of parameter values corresponding to uncontrollable systems (an uncontrollability set) is studied. The typical (generic) structure of the uncontrollability set is described. A constructive method of perturbation analysis of the uncontrollability set is developed. Formulae of first-order approximations for the uncontrollability set and generalized eigenvalues (uncontrollable modes) are derived and used for numerical construction of the uncontrollability set. The method is based on the versal deformation theory for matrix pairs under feedback equivalence. As an example, the uncontrollability set is found for a three-parameter two-degree-of-freedom mechanical system.
\end{abstract}

Key words. uncontrollability, feedback equivalence, uncontrollable mode, perturbation, matrix pair, versal deformation

AMS subject classification. 93B

DOI. $10.1137 /$ S0363012902405339

1. Introduction. The concept of controllability is very important in the study of control problems. It describes the possibility of transferring a system to a required state using a given set of input (control) variables. Uncontrollability makes operation of a system in the whole state space impossible and signals the fundamental trouble with a control problem or underlying physical system. Similar difficulties appear for nearly uncontrollable systems, which require big control resources for control performance and which are strongly affected by imperfections and disturbances of the system.

The well-developed control theory exists for linear dynamical systems [3, 13]. Nevertheless, there are essential problems in using classical controllability criteria for numerical implementation. This is related to the structural instability of an uncontrollable system, which becomes controllable under an arbitrarily small perturbation. In this respect, it is important to know how far our system is from the nearest uncontrollable one. This problem was studied by many authors; see $[2,10,11,12]$ and references therein.

Design of a particular control system requires checking the controllability condition for different values of parameters. In this analysis, the knowledge on the structure of the uncontrollability set (a set of parameter values corresponding to uncontrollable systems) is very useful and helps in avoiding the dangerous nearness to uncontrollability. In this paper, basic qualitative properties of the uncontrollability set for a generic (typical) multi-input linear dynamical system depending on several parameters are investigated. This includes a description of a regular part of the uncontrollability set and its basic singularities. Then the quantitative perturbation method for local analysis of the uncontrollability set near its regular points is developed. Application

\footnotetext{
*Received by the editors April 11, 2002; accepted for publication (in revised form) April 1, 2003; published electronically October 2, 2003. This work was supported by Russian Foundation of Basic Research grant 02-01-39004.

http://www.siam.org/journals/sicon/42-4/40533.html

†Institute of Mechanics, Moscow State Lomonosov University, Michurinsky pr. 1, 119192 Moscow, Russia (mailybaev@imec.msu.ru).
} 
of this method to numerical calculation of a regular part of the uncontrollability set in the parameter space is proposed. As an example, an elastic mechanical system controlled by a force and dependent on three design parameters is studied. The results of the paper are based on the versal deformation theory for matrix pairs under state feedback equivalence $[6,8,14]$.

The paper is organized as follows. Section 2 describes basic concepts and results of the mathematical control theory. Section 3 studies the qualitative structure of the uncontrollability set. Quantitative perturbation method for local analysis of the uncontrollability set is developed in section 4 . In section 5, a numerical method for computation of a regular part of the uncontrollability set is constructed and applied to the analysis of a specific mechanical system. Section 6 is devoted to singularities of the uncontrollability set. The conclusion summarizes the contribution.

2. Controllability, feedback equivalence, and versal deformation. Let us consider a dynamical system described by the system of linear ordinary differential equations

$$
\dot{\mathbf{x}}(t)=\mathbf{A} \mathbf{x}(t)+\mathbf{B u}(t),
$$

where $\mathbf{x} \in \mathbb{R}^{n}$ is a state vector, $\mathbf{u} \in \mathbb{R}^{m}$ is an input vector, and $\mathbf{A}$ and $\mathbf{B}$ are $n \times n$ and $n \times m$ real time-invariant matrices, respectively; the dot denotes differentiation with respect to time $t$. System (2.1) is called (state) controllable if it is possible to construct a control signal $\mathbf{u}(t)$ that will transfer an initial state to any final state in finite time $[3,13]$. Otherwise, the system is said to be uncontrollable. The classical criterion of controllability says that the system is controllable if and only if the $n \times n m$ controllability matrix $\mathbf{C}=\left[\mathbf{B}, \mathbf{A B}, \ldots, \mathbf{A}^{n-1} \mathbf{B}\right]$ has full rank $[3,13]$

$$
\operatorname{rank}\left[\mathbf{B}, \mathbf{A B}, \ldots, \mathbf{A}^{n-1} \mathbf{B}\right]=n \text {. }
$$

System (2.1) is determined by a matrix pair $(\mathbf{A}, \mathbf{B})$. Let us denote the set of all matrix pairs by $\mathcal{M}=\left\{(\mathbf{A}, \mathbf{B}) \mid \mathbf{A} \in \mathbb{R}^{n \times n}, \mathbf{B} \in \mathbb{R}^{n \times m}\right\}$. We will use the short notation $\alpha=(\mathbf{A}, \mathbf{B})$ for a matrix pair. The matrix pair $\alpha$ is called controllable (or uncontrollable) if the corresponding system (2.1) is controllable (or uncontrollable).

2.1. Feedback equivalence. Let us apply a linear feedback transformation of the input vector and perform a change of basis in the state and input spaces. The new state vector $\overline{\mathbf{x}}$ and input vector $\overline{\mathbf{u}}$ are related to $\mathbf{x}$ and $\mathbf{u}$ by the expressions

$$
\mathbf{x}=\mathbf{P} \overline{\mathbf{x}}, \quad \mathbf{u}=\mathbf{Q} \overline{\mathbf{u}}+\mathbf{R} \overline{\mathbf{x}},
$$

where $\mathbf{P}$ and $\mathbf{Q}$ are $n \times n$ and $m \times m$ nonsingular real matrices; $\mathbf{R}$ is an $m \times n$ real matrix. The substitution of (2.3) into (2.1) yields

$$
\dot{\overline{\mathbf{x}}}(t)=\overline{\mathbf{A}} \overline{\mathbf{x}}(t)+\overline{\mathbf{B}} \overline{\mathbf{u}}(t),
$$

where

$$
\overline{\mathbf{A}}=\mathbf{P}^{-1}(\mathbf{A P}+\mathbf{B R}), \quad \overline{\mathbf{B}}=\mathbf{P}^{-1} \mathbf{B Q} .
$$

Systems (2.1) and (2.4) (as well as the corresponding matrix pairs $\alpha=(\mathbf{A}, \mathbf{B})$ and $\bar{\alpha}=(\overline{\mathbf{A}}, \overline{\mathbf{B}})$ ) are called feedback equivalent (also called block equivalent) $[9,13]$. The controllability property is invariant under the feedback equivalence transformation. 
Let us introduce the short notation $\bar{\alpha}=\gamma \circ \alpha$ for the feedback equivalence transformation (2.5) applied to a matrix pair $\alpha=(\mathbf{A}, \mathbf{B})$, where $\gamma$ denotes a triple $\gamma=(\mathbf{P}, \mathbf{Q}, \mathbf{R})$. We denote the set of all feedback equivalence transformations by $\mathcal{G}=\left\{(\mathbf{P}, \mathbf{Q}, \mathbf{R}) \mid \mathbf{P} \in \mathbb{R}^{n \times n}, \mathbf{Q} \in \mathbb{R}^{m \times m}, \mathbf{R} \in \mathbb{R}^{m \times n}, \operatorname{det} \mathbf{P} \neq 0, \operatorname{det} \mathbf{Q} \neq 0\right\}$. Note that relations (2.5) determine the Lie group structure in $\mathcal{G}$ with the multiplication and inversion of elements given by

$$
\begin{gathered}
\gamma_{1} \gamma_{2}=\left(\mathbf{P}_{1} \mathbf{P}_{2}, \mathbf{Q}_{1} \mathbf{Q}_{2}, \mathbf{R}_{1} \mathbf{P}_{2}+\mathbf{Q}_{1} \mathbf{R}_{2}\right) \in \mathcal{G}, \quad \gamma_{i}=\left(\mathbf{P}_{i}, \mathbf{Q}_{i}, \mathbf{R}_{i}\right) \in \mathcal{G}, \\
\gamma^{-1}=\left(\mathbf{P}^{-1}, \mathbf{Q}^{-1},-\mathbf{Q}^{-1} \mathbf{R} \mathbf{P}^{-1}\right) \in \mathcal{G}, \quad \gamma=(\mathbf{P}, \mathbf{Q}, \mathbf{R}) \in \mathcal{G}
\end{gathered}
$$

such that $\gamma_{1} \gamma_{2} \circ \alpha=\gamma_{2} \circ\left(\gamma_{1} \circ \alpha\right)$ and $\gamma \gamma^{-1} \circ \alpha=\alpha$ for any $\alpha \in \mathcal{M}$. The unit element of $\mathcal{G}$ is $e=\left(\mathbf{I}_{n}, \mathbf{I}_{m}, 0\right)$, where $\mathbf{I}_{n}$ and $\mathbf{I}_{m}$ are $n \times n$ and $m \times m$ identity matrices, respectively. This triple has the property $\alpha=e \circ \alpha$ for any pair $\alpha \in \mathcal{M}$.

2.2. Equivalence classes and their local structure. Let us consider a fixed matrix pair $\alpha_{0}=\left(\mathbf{A}_{0}, \mathbf{B}_{0}\right) \in \mathcal{M}$. A set of all pairs $\alpha$ feedback equivalent to $\alpha_{0}$ is called the orbit of $\alpha_{0}$ and denoted by

$$
\mathcal{O}\left(\alpha_{0}\right)=\left\{\alpha \in \mathcal{M} \mid \alpha=\gamma \circ \alpha_{0}, \gamma \in \mathcal{G}\right\} .
$$

The orbit is a smooth submanifold of $\mathcal{M}$.

The orbit $\mathcal{O}\left(\alpha_{0}\right)$ can be represented by its arbitrary member $\alpha \in \mathcal{O}\left(\alpha_{0}\right)$. Therefore, to describe the orbit it is convenient to choose a pair $\alpha$ having, in some sense, the simplest form. One such form, called a Brunovsky canonical form, is represented by the matrix pair $[9,13]$

$$
\alpha_{b}=\left(\mathbf{A}_{b}, \mathbf{B}_{b}\right), \quad \mathbf{A}_{b}=\left(\begin{array}{cc}
\mathbf{N} & 0 \\
0 & \mathbf{J}
\end{array}\right), \quad \mathbf{B}_{b}=\left(\begin{array}{cc}
\mathbf{E} & 0 \\
0 & 0
\end{array}\right),
$$

where $\mathbf{J}$ is the real Jordan canonical form (real counterpart of the Jordan form); $\mathbf{N}=\operatorname{diag}\left(\mathbf{N}_{1}, \ldots, \mathbf{N}_{r}\right) ; \mathbf{E}=\operatorname{diag}\left(\mathbf{E}_{1}, \ldots, \mathbf{E}_{r}\right) ; \mathbf{N}_{i}$ and $\mathbf{E}_{i}$ are $k_{i} \times k_{i}$ and $k_{i} \times 1$ matrices, respectively, having the form

$$
\mathbf{N}_{i}=\left(\begin{array}{cccc}
0 & 1 & & \\
& 0 & \ddots & \\
& & \ddots & 1 \\
& & & 0
\end{array}\right), \quad \mathbf{E}_{i}=\left(\begin{array}{c}
0 \\
\vdots \\
0 \\
1
\end{array}\right) .
$$

The numbers $k_{1} \geq \cdots \geq k_{r}>0$ are called controllability indices or Kronecker indices of the system; $\mathbf{N}$ is called a Kronecker part and $\mathbf{J}$ is called a Jordan part of the Brunovsky form. Any matrix pair $\alpha \in \mathcal{M}$ is feedback equivalent to the corresponding Brunovsky form, which is unique up to the permutation of blocks in the Jordan matrix J. Using condition (2.2), one can check that the matrix pair $\alpha$ is controllable if and only if its Brunovsky form has no Jordan part. If the pair is uncontrollable, then eigenvalues of the Jordan part $\mathbf{J}$ are called generalized eigenvalues or uncontrollable modes.

Let us introduce the function $f_{\alpha_{0}}(\gamma)=\gamma \circ \alpha_{0}$, which is a smooth function from $\mathcal{G}$ to $\mathcal{M}$. Then the orbit $\mathcal{O}\left(\alpha_{0}\right)$ can be seen as the range of the mapping $f_{\alpha_{0}}$, i.e.,

$$
\mathcal{O}\left(\alpha_{0}\right)=\operatorname{Im} f_{\alpha_{0}}
$$


Let us denote by $T_{e} \mathcal{G}$ the tangent space to $\mathcal{G}$ at the unit element $e$. Since $\mathcal{G}$ is an open set, we have $T_{e} \mathcal{G}=\left\{(\mathbf{U}, \mathbf{V}, \mathbf{W}) \mid \mathbf{U} \in \mathbb{R}^{n \times n}, \mathbf{V} \in \mathbb{R}^{m \times m}, \mathbf{W} \in \mathbb{R}^{m \times n}\right\}$. The linear spaces $\mathcal{M}$ and $T_{e} \mathcal{G}$ are equipped with the Euclidean scalar products

$$
\begin{aligned}
& \left\langle\alpha_{1}, \alpha_{2}\right\rangle_{1}=\operatorname{trace}\left(\mathbf{A}_{1} \mathbf{A}_{2}^{T}\right)+\operatorname{trace}\left(\mathbf{B}_{1} \mathbf{B}_{2}^{T}\right), \\
& \left\langle\xi_{1}, \xi_{2}\right\rangle_{2}=\operatorname{trace}\left(\mathbf{U}_{1} \mathbf{U}_{2}^{T}\right)+\operatorname{trace}\left(\mathbf{V}_{1} \mathbf{V}_{2}^{T}\right)+\operatorname{trace}\left(\mathbf{W}_{1} \mathbf{W}_{2}^{T}\right),
\end{aligned}
$$

where $\alpha_{i}=\left(\mathbf{A}_{i}, \mathbf{B}_{i}\right) \in \mathcal{M}, \xi_{i}=\left(\mathbf{U}_{i}, \mathbf{V}_{i}, \mathbf{W}_{i}\right) \in T_{e} \mathcal{G}$, and $\mathbf{A}^{T}$ denotes the transposed matrix. Let us introduce linear mappings $d f_{\alpha_{0}}: T_{e} \mathcal{G} \longrightarrow \mathcal{M}$ and $d f_{\alpha_{0}}^{*}: \mathcal{M} \longrightarrow T_{e} \mathcal{G}$ as follows:

$$
\begin{array}{ll}
d f_{\alpha_{0}}(\xi)=\left(\mathbf{A}_{0} \mathbf{U}-\mathbf{U} \mathbf{A}_{0}+\mathbf{B}_{0} \mathbf{W}, \mathbf{B}_{0} \mathbf{V}-\mathbf{U B}_{0}\right), & \xi=(\mathbf{U}, \mathbf{V}, \mathbf{W}), \\
d f_{\alpha_{0}}^{*}(\alpha)=\left(\mathbf{A}_{0}^{T} \mathbf{A}-\mathbf{A} \mathbf{A}_{0}^{T}-\mathbf{B B}_{0}^{T}, \mathbf{B}_{0}^{T} \mathbf{B}, \mathbf{B}_{0}^{T} \mathbf{A}\right), & \alpha=(\mathbf{A}, \mathbf{B}) .
\end{array}
$$

It is straightforward to check that $d f_{\alpha_{0}}$ is the differential of the function $f_{\alpha_{0}}$ at the unit element $e=\left(\mathbf{I}_{n}, \mathbf{I}_{m}, 0\right)[6]$, and $d f_{\alpha_{0}}^{*}$ is the adjoint function defined by the relation [7]

$$
\left\langle d f_{\alpha_{0}}(\xi), \alpha\right\rangle_{1}=\left\langle\xi, d f_{\alpha_{0}}^{*}(\alpha)\right\rangle_{2}, \quad \alpha \in \mathcal{M}, \quad \xi \in T_{e} \mathcal{G} .
$$

A local structure of the orbit $\mathcal{O}\left(\alpha_{0}\right)$ near the point $\alpha_{0}$ is determined by the range of the mapping $d f_{\alpha_{0}}$ and null-space of $d f_{\alpha_{0}}^{*}$ as follows [8]:

$$
\begin{gathered}
T_{\alpha_{0}} \mathcal{O}\left(\alpha_{0}\right)=\operatorname{Im} d f_{\alpha_{0}}, \\
\left(T_{\alpha_{0}} \mathcal{O}\left(\alpha_{0}\right)\right)^{\perp}=\operatorname{Ker} d f_{\alpha_{0}}^{*},
\end{gathered}
$$

where $T_{\alpha_{0}} \mathcal{O}\left(\alpha_{0}\right)$ is the tangent space to $\mathcal{O}\left(\alpha_{0}\right)$ at the point $\alpha_{0} ;\left(T_{\alpha_{0}} \mathcal{O}\left(\alpha_{0}\right)\right)^{\perp}$ denotes the normal complimentary subspace to $T_{\alpha_{0}} \mathcal{O}\left(\alpha_{0}\right)$ in $\mathcal{M}$. In addition, we denote by $\left(T_{\alpha_{0}} \mathcal{O}\left(\alpha_{0}\right)\right)^{c}$ an arbitrary complimentary subspace to $T_{\alpha_{0}} \mathcal{O}\left(\alpha_{0}\right)$ in $\mathcal{M}$.

2.3. Versal deformation. A multiparameter dynamical system (2.1) is described by a matrix pair $\alpha(\mathbf{p})$ smoothly dependent on a vector of parameters $\mathbf{p}=$ $\left(p_{1}, \ldots, p_{k}\right) \in \mathbb{R}^{k}$. The function $\alpha(\mathbf{p})$ is called the family of matrix pairs. The family $\alpha(\mathbf{p})$ determined in the neighborhood of a point $\mathbf{p}_{0}$ is called a deformation of the matrix pair $\alpha_{0}=\alpha\left(\mathbf{p}_{0}\right)$. Using feedback equivalence transformation $\gamma(\mathbf{p}) \circ \alpha(\mathbf{p})$, the family $\alpha(\mathbf{p})$ can be reduced to a more simple form. Nevertheless, a reduction to the Brunovsky form generally cannot be achieved by the feedback equivalence transformation $\gamma(\mathbf{p})$ smoothly dependent on parameters. The following theorem proved in $[6,8]$ provides another form called a versal deformation that can be used for multiparameter families of matrix pairs. Note that the concept of versal deformation was first introduced by Arnold [1] for families of square complex matrices; see also [14] for the generalization to the case of a Lie group acting on a complex manifold.

THEOREM 2.1. Let $\alpha(\mathbf{p})$ be a family of matrix pairs. Then in the neighborhood of a point $\mathbf{p}_{0}$, the family $\alpha(\mathbf{p})$ can be represented in the form

$$
\alpha(\mathbf{p})=\gamma(\mathbf{p}) \circ\left(\alpha_{0}+\sum_{i=1}^{\ell} q_{i}(\mathbf{p}) \alpha_{i}^{c}\right) .
$$

In this formula $\left\{\alpha_{1}^{c}, \ldots, \alpha_{\ell}^{c}\right\}, \ell=\operatorname{dim}\left(T_{\alpha_{0}} \mathcal{O}\left(\alpha_{0}\right)\right)^{c}$, is a basis of $\left(T_{\alpha_{0}} \mathcal{O}\left(\alpha_{0}\right)\right)^{c} ; \gamma(\mathbf{p})$ is a feedback equivalence transformation smoothly dependent on $\mathbf{p}$ such that $\gamma\left(\mathbf{p}_{0}\right)=e$; $q_{1}(\mathbf{p}), \ldots, q_{\ell}(\mathbf{p})$ are smooth functions, whose values and derivatives at $\mathbf{p}_{0}$ are

$$
q_{1}\left(\mathbf{p}_{0}\right)=\cdots=q_{\ell}\left(\mathbf{p}_{0}\right)=0,
$$




$$
\left(\begin{array}{c}
\frac{\partial q_{1}}{\partial p_{j}} \\
\vdots \\
\frac{\partial q_{\ell}}{\partial p_{j}}
\end{array}\right)=\mathbf{Z}^{-1}\left(\begin{array}{c}
\left\langle\frac{\partial \alpha}{\partial p_{j}}, \alpha_{1}^{n}\right\rangle_{1} \\
\vdots \\
\left\langle\frac{\partial \alpha}{\partial p_{j}}, \alpha_{\ell}^{n}\right\rangle_{1}
\end{array}\right)
$$

where $\left\{\alpha_{1}^{n}, \ldots, \alpha_{\ell}^{n}\right\}$ is a basis of the linear space $\left(T_{\alpha_{0}} \mathcal{O}\left(\alpha_{0}\right)\right)^{\perp} ; \mathbf{Z}$ is a nonsingular $\ell \times \ell$ matrix with the elements $z_{i j}=\left\langle\alpha_{j}^{c}, \alpha_{i}^{n}\right\rangle_{1}$.

Formulae for derivatives of the functions $q_{1}(\mathbf{p}), \ldots, q_{\ell}(\mathbf{p})$ and $\gamma(\mathbf{p})$ of any order were derived in [8]. The family of matrix pairs

$$
\beta(\mathbf{q})=\alpha_{0}+\sum_{i=1}^{\ell} q_{i} \alpha_{i}^{c}, \quad \mathbf{q}=\left(q_{1}, \ldots, q_{\ell}\right),
$$

is called a versal deformation of the matrix pair $\alpha_{0} ; \mathbf{q}$ is a parameter vector of the versal deformation [1]. The main idea of the above theorem is that any matrix family $\alpha(\mathbf{p})$ with a given pair $\alpha_{0}=\alpha\left(\mathbf{p}_{0}\right)$ can be transformed locally to the versal deformation $\beta(\mathbf{q})$, which has an explicit and simple form, by the feedback equivalence transformation $\gamma(\mathbf{p})$ smoothly dependent on $\mathbf{p}$ and smooth change of parameters $\mathbf{q}=\mathbf{q}(\mathbf{p})$. Note that the bases $\left\{\alpha_{1}^{c}, \ldots, \alpha_{\ell}^{c}\right\}$ and $\left\{\alpha_{1}^{n}, \ldots, \alpha_{\ell}^{n}\right\}$ have been found explicitly in [6] for matrix pairs reduced to the Brunovsky canonical form.

Example 2.1. Let us consider a one-parameter family of matrix pairs $\alpha(p)=$ $(\mathbf{A}(p), \mathbf{B}(p))$, where

$$
\mathbf{A}(p)=\left(\begin{array}{ccc}
p & 0 & p^{2} \\
2 p & p & -p \\
3 p & p & 2+p^{3}
\end{array}\right), \quad \mathbf{B}(p)=\left(\begin{array}{cc}
1 & p \\
p & 1 \\
0 & -p
\end{array}\right)
$$

Family (2.20) determines a one-parameter dynamical system (2.1) with three-dimensional state space and two-dimensional input vector. The pair $\alpha_{0}=\alpha\left(p_{0}\right)$ for $p_{0}=0$ has the form

$$
\alpha_{0}=\left(\left(\begin{array}{ccc}
0 & 0 & 0 \\
0 & 0 & 0 \\
0 & 0 & 2
\end{array}\right),\left(\begin{array}{ll}
1 & 0 \\
0 & 1 \\
0 & 0
\end{array}\right)\right),
$$

which is the Brunovsky canonical form (2.8), (2.9) with $r=2, k_{1}=k_{2}=1$, and $\mathbf{J}=(2)$. Solving the linear equation $d f_{\alpha_{0}}^{*}(\alpha)=0$ with respect to $\alpha$ and using relation (2.15), we find that the space $\left(T_{\alpha_{0}} \mathcal{O}\left(\alpha_{0}\right)\right)^{\perp}$ has dimension $\ell=3$ and consists of the matrix pairs

$$
\left(\left(\begin{array}{ccc}
0 & 0 & 0 \\
0 & 0 & 0 \\
q_{1} & q_{2} & q_{3}
\end{array}\right),\left(\begin{array}{cc}
0 & 0 \\
0 & 0 \\
2 q_{1} & 2 q_{2}
\end{array}\right)\right) \in\left(T_{\alpha_{0}} \mathcal{O}\left(\alpha_{0}\right)\right)^{\perp}, \quad q_{1}, q_{2}, q_{3} \in \mathbb{R} .
$$

The complimentary subspace $\left(T_{\alpha_{0}} \mathcal{O}\left(\alpha_{0}\right)\right)^{c}$ can be chosen in a more simple form as follows:

$$
\left(\left(\begin{array}{ccc}
0 & 0 & 0 \\
0 & 0 & 0 \\
q_{1} & q_{2} & q_{3}
\end{array}\right),\left(\begin{array}{cc}
0 & 0 \\
0 & 0 \\
0 & 0
\end{array}\right)\right) \in\left(T_{\alpha_{0}} \mathcal{O}\left(\alpha_{0}\right)\right)^{c}, \quad q_{1}, q_{2}, q_{3} \in \mathbb{R} .
$$


Expressions (2.22) and (2.23) generate the bases $\left\{\alpha_{1}^{n}, \alpha_{2}^{n}, \alpha_{3}^{n}\right\}$ and $\left\{\alpha_{1}^{c}, \alpha_{2}^{c}, \alpha_{3}^{c}\right\}$ as coefficients corresponding to $q_{1}, q_{2}, q_{3}$.

By Theorem 2.1, family (2.20) can be represented in the form $\alpha(p)=\gamma(p) \circ$ $\beta(\mathbf{q}(p))$, where $\beta(\mathbf{q})$ is the versal deformation

$$
\beta(\mathbf{q})=\left(\left(\begin{array}{ccc}
0 & 0 & 0 \\
0 & 0 & 0 \\
q_{1} & q_{2} & 2+q_{3}
\end{array}\right),\left(\begin{array}{cc}
1 & 0 \\
0 & 1 \\
0 & 0
\end{array}\right)\right), \quad \mathbf{q}=\left(q_{1}, q_{2}, q_{3}\right),
$$

$\gamma(p)$ is a feedback equivalence transformation smoothly dependent on $p$, and $\mathbf{q}=\mathbf{q}(p)$ is a smooth change of parameters. Derivatives of the functions $q_{1}(p), q_{2}(p), q_{3}(p)$ at $p_{0}=0$ can be calculated by expression (2.18) as follows:

$$
\frac{d q_{1}}{d p}=3, \quad \frac{d q_{2}}{d p}=-1, \quad \frac{d q_{3}}{d p}=0
$$

3. Structure of the uncontrollability set. Let us consider a multiparameter dynamical system described by a family of matrix pairs $\alpha(\mathbf{p})$. A set of values of the parameter vector $\mathbf{p}$ such that $\alpha(\mathbf{p})$ is uncontrollable is called the uncontrollability set and denoted by

$$
\mathcal{N}=\{\mathbf{p} \mid \alpha(\mathbf{p}) \text { is uncontrollable }\} .
$$

It is known that $\mathcal{N}$ is typically a set of zero measure [13]. In particular, any uncontrollable system can be made controllable by an arbitrarily small perturbation of the matrices $\mathbf{A}$ and $\mathbf{B}$. Nevertheless, when the parameter vector is close to the uncontrollability set $\mathcal{N}$, the system becomes practically uncontrollable due to small perturbations and uncertainties existing in every physical system, and the necessity of using big control resources (large $\|\mathbf{u}\|$ ) or long time for control operation. This makes analysis and construction of the uncontrollability set in the parameter space important for the design of control systems.

As was mentioned in the previous section, for a fixed value of $\mathbf{p}$ the matrix pair $\alpha(\mathbf{p})$ can be transformed to the Brunovsky canonical form (2.8), and the uncontrollability of $\alpha(\mathbf{p})$ is equivalent to the existence of the Jordan part in this form. Let us consider two specific types of the Brunovsky form that will be important for further analysis. These two forms are represented by the $1 \times 1$ and $2 \times 2$ Jordan parts

$$
\mathbf{J}_{\sigma}=(\sigma), \quad \mathbf{J}_{\sigma \pm i \omega}=\left(\begin{array}{cc}
\sigma & \omega \\
-\omega & \sigma
\end{array}\right), \quad \sigma, \omega \in \mathbb{R}, \omega>0,
$$

corresponding to a real simple eigenvalue $\sigma$ and a pair of complex conjugate simple eigenvalues $\sigma \pm i \omega$, respectively. Structure of the Kronecker part can be arbitrary. We refer to matrix pairs having the described structures of the Brunovsky form as pairs of $\mathbf{J}_{\sigma}$ and $\mathbf{J}_{\sigma \pm i \omega}$ types.

To describe the qualitative structure of the uncontrollability set $\mathcal{N}$, it is reasonable to restrict our attention to the generic (typical) situation. This corresponds to a typical form of the set $\mathcal{N}$ such that small perturbations of the family $\alpha(\mathbf{p})$ do not lead to qualitative changes in the geometry and structure of $\mathcal{N}$ but result only in its small shift in the parameter space. For more precise mathematical formulation of the concept "generic," see [1]. Consideration of the generic case allows extracting the most typical and interesting information on the structure of the uncontrollability 


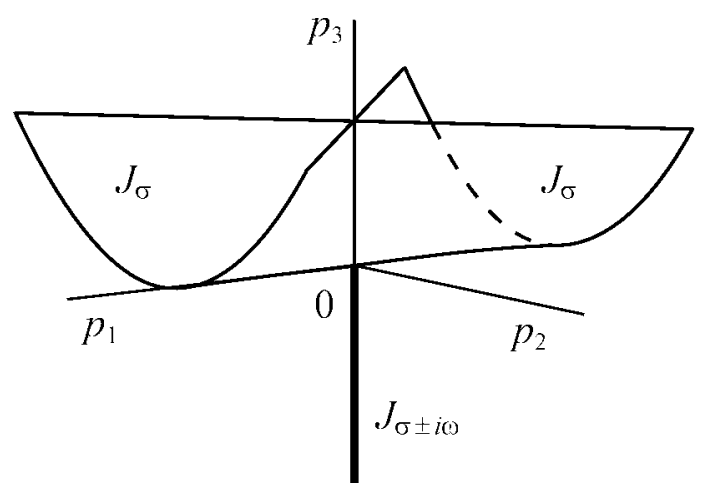

FIG. 3.1. Structure of the uncontrollability set.

set, which is valid for almost all multi-input linear dynamical systems dependent on parameters. The following theorem provides the basic qualitative description of this set.

THEOREM 3.1. In the generic case the uncontrollability set $\mathcal{N}$ of the family of matrix pairs $\alpha(\mathbf{p})$ has a regular part, which consists of smooth surfaces of codimensions $m$ and $2 m$ corresponding to matrix pairs of $\mathbf{J}_{\sigma}$ and $\mathbf{J}_{\sigma \pm i \omega}$ types, respectively. Points $\mathbf{p} \in \mathcal{N}$ such that the matrix pair $\alpha(\mathbf{p})$ has a different type of the Brunovsky form belong to the boundary of these surfaces.

Example 3.1. Let us consider the family of matrix pairs

$$
\alpha(\mathbf{p})=\left(\left(\begin{array}{cccc}
0 & 1 & 0 & 0 \\
0 & 0 & 0 & 0 \\
p_{1} & 0 & 0 & 1 \\
p_{2} & 0 & p_{3} & 0
\end{array}\right),\left(\begin{array}{l}
0 \\
1 \\
0 \\
0
\end{array}\right)\right), \quad \mathbf{p}=\left(p_{1}, p_{2}, p_{3}\right) .
$$

Using the controllability condition (2.2), we find the uncontrollability set in the form

$$
\mathcal{N}=\left\{\mathbf{p} \in \mathbb{R}^{3} \mid p_{1}^{2} p_{3}-p_{2}^{2}=0\right\} .
$$

This set is shown in Figure 3.1. It represents the well-known Whitney-Cayley umbrella [1]. The structure of $\mathcal{N}$ agrees with Theorem 3.1. Indeed, the set $\mathcal{N}$ has a "handle" (the ray $p_{1}=p_{2}=0, p_{3}<0$ ), which has codimension $2 m=2$ and corresponds to pairs of $\mathbf{J}_{\sigma \pm i \omega}$ type with the generalized eigenvalues $\sigma \pm i \omega= \pm i \sqrt{-p_{3}}$. There are two smooth surfaces of codimension $m=1$ determined by the equation $p_{1}^{2} p_{3}-p_{2}^{2}=0$ for $p_{3} \geq 0, p_{1}<0$ and $p_{3} \geq 0, p_{1}>0$, which correspond to the pairs of $\mathbf{J}_{\sigma}$ type; the generalized eigenvalue is $\sigma=-p_{2} / p_{1}$. The "handle" and two surfaces form a regular part of $\mathcal{N}$. There are also different types of uncontrollable pairs: at the point $\mathbf{p}=0$ we have a pair with the Jordan part $\mathbf{J}=\left(\begin{array}{ll}0 & 1 \\ 0 & 0\end{array}\right)$, and points of the ray $p_{1}=p_{2}=0, p_{3}>0$ correspond to pairs having two different real generalized eigenvalues $\sigma_{1,2}= \pm \sqrt{p_{3}}$. These points determine singularities of $\mathcal{N}$ and belong to the boundary of the regular part.

According to Theorem 3.1, we can expect to find a similar structure of the uncontrollability set for almost all families of matrix pairs. This means that for the construction of the uncontrollability set it is sufficient to find its regular part, i.e., points of $\mathbf{J}_{\sigma}$ and $\mathbf{J}_{\sigma \pm i \omega}$ types forming smooth surfaces of codimensions $m$ and $2 m$, respectively. Then the whole set $\mathcal{N}$ will be the closure of these surfaces. 
Proof. The proof is based on general results of the singularity theory and uses versal deformations. In order to avoid special mathematical language, we describe general steps and ideas of the proof, while the details can be easily reconstructed by the reader using the cited literature.

The decomposition of the parameter space into subsets (strata) according to the Brunovsky structure of the corresponding matrix pair $\alpha(\mathbf{p})$ is called the bifurcation diagram. The singularity theory says that the qualitative local structure of the bifurcation diagram (and, hence, of the uncontrollability set) for a generic family $\alpha(\mathbf{p})$ is the same as for the versal deformation $\beta(\mathbf{q})$ [1]. In particular, codimensions of strata in the corresponding parameter spaces coincide. This follows from the relation $\alpha(\mathbf{p})=\gamma(\mathbf{p}) \circ \beta(\mathbf{q}(\mathbf{p}))$ and the property that the Brunovsky canonical form is invariant under the feedback equivalence transformation $\gamma(\mathbf{p})$.

Let us consider a matrix pair $\alpha_{0}=\alpha\left(\mathbf{p}_{0}\right)$. Without loss of generality, we can assume that $\alpha_{0}$ is transformed to the Brunovsky canonical form (2.8). Then its versal deformation can be taken in the form

$$
\beta(\mathbf{q})=\left(\left(\begin{array}{cc}
\mathbf{N} & 0 \\
\mathbf{X}_{21}^{c} & \mathbf{J}+\mathbf{X}_{22}^{c}
\end{array}\right),\left(\begin{array}{cc}
\mathbf{E}+\mathbf{Y}_{11}^{c} & \mathbf{Y}_{12}^{c} \\
0 & \mathbf{Y}_{22}^{c}
\end{array}\right)\right)
$$

where $\mathbf{X}_{i j}^{c}(\mathbf{q})$ and $\mathbf{Y}_{i j}^{c}(\mathbf{q})$ are matrices depending on a vector of parameters $\mathbf{q} \in \mathbb{R}^{\ell}$ such that every matrix is zero at $\mathbf{q}=0$. Explicit form of these matrices depends on the structure of $\mathbf{N}$ and $\mathbf{J}$. In the case $\mathbf{J}=\mathbf{J}_{\sigma}$ we can take [6]

$$
\mathbf{X}_{21}^{c}=\left(\mathbf{L}_{1}^{c}\left(q_{1}\right), \ldots, \mathbf{L}_{r}^{c}\left(q_{r}\right)\right), \mathbf{Y}_{22}^{c}=\left(q_{r+1}, \ldots, q_{m}\right), \mathbf{J}+\mathbf{X}_{22}^{c}=\left(\sigma+q_{m+1}\right),
$$

where $\mathbf{L}_{i}^{c}\left(q_{i}\right)=\left(q_{i}, 0, \ldots, 0\right)$ is a $1 \times k_{i}$ matrix; $k_{1}, \ldots, k_{r}$ are the controllability indices of the Kronecker part $\mathbf{N}$. The matrices $\mathbf{Y}_{11}^{c}$ and $\mathbf{Y}_{12}^{c}$ depend on $q_{m+2}, \ldots, q_{\ell}$. Using the controllability condition (2.2), one can show that in the vicinity of $\mathbf{q}=0$ versal deformation (3.5), (3.6) is controllable if and only if at least one of the parameters $q_{1}, \ldots, q_{m}$ is nonzero. Hence, the uncontrollability set for the versal deformation is given locally by the equalities

$$
q_{1}=\cdots=q_{m}=0
$$

Taking a point $\mathbf{q}$ such that (3.7) holds, it is easy to see that the pair $\beta(\mathbf{q})$ has $\mathbf{J}_{\sigma}$ type with the generalized eigenvalue $\sigma+q_{m+1}$. Since the uncontrollability set of a generic family of matrix pairs $\alpha(\mathbf{p})$ has the same local structure, we conclude that the set $\mathcal{N}$ in the neighborhood of a point $\mathbf{p}_{0}$, corresponding to a pair of $\mathbf{J}_{\sigma}$ type, is a smooth surface of codimension $m$, whose points correspond to pairs of $\mathbf{J}_{\sigma}$ type.

Analogously, in the case $\mathbf{J}=\mathbf{J}_{\sigma \pm i \omega}$ we can take [6]

$$
\begin{aligned}
\mathbf{X}_{21}^{c}= & \left(\mathbf{L}_{1}^{c}\left(q_{1}, q_{2}\right), \ldots, \mathbf{L}_{r}^{c}\left(q_{2 r-1}, q_{2 r}\right)\right), \\
\mathbf{Y}_{22}^{c} & =\left(\begin{array}{ccc}
q_{2 r+1} & \cdots & q_{2 m-1} \\
q_{2 r+2} & \cdots & q_{2 m}
\end{array}\right), \\
\mathbf{J}+\mathbf{X}_{22}^{c} & =\left(\begin{array}{cc}
\sigma+q_{2 m+1} & \omega+q_{2 m+2} \\
-\omega-q_{2 m+2} & \sigma+q_{2 m+1}
\end{array}\right),
\end{aligned}
$$

where $\mathbf{L}_{i}^{c}\left(q_{2 i-1}, q_{2 i}\right)$ is a $2 \times k_{i}$ matrix of the form

$$
\mathbf{L}_{i}^{c}\left(q_{2 i-1}, q_{2 i}\right)=\left(\begin{array}{cccc}
q_{2 i-1} & 0 & \cdots & 0 \\
q_{2 i} & 0 & \cdots & 0
\end{array}\right) .
$$


The matrices $\mathbf{Y}_{11}^{c}$ and $\mathbf{Y}_{12}^{c}$ depend on $q_{2 m+3}, \ldots, q_{\ell}$. The uncontrollability set of this versal deformation in the vicinity of $\mathbf{q}=0$ is given by the equations

$$
q_{1}=\cdots=q_{2 m}=0 .
$$

At the points $\mathbf{q}$ satisfying (3.10), the pair $\beta(\mathbf{q})$ has $\mathbf{J}_{\sigma \pm i \omega}$ type, and the complex conjugate generalized eigenvalues are equal to $\sigma+q_{2 m+1} \pm i\left(\omega+q_{2 m+2}\right)$. Hence, the uncontrollability set of a generic family $\alpha(\mathbf{p})$ in the neighborhood of a point $\mathbf{p}_{0}$, corresponding to a pair of $\mathbf{J}_{\sigma \pm i \omega}$ type, is a smooth surface of codimension $2 \mathrm{~m}$. Points of this surface correspond to matrix pairs of $\mathbf{J}_{\sigma \pm i \omega}$ type.

Now, let us consider a matrix pair $\alpha_{0}$ having a different Jordan part $\mathbf{J}$. In this case the block $\mathbf{J}+\mathbf{X}_{22}^{c}$ is identical to the versal deformation of a square matrix $\mathbf{J}$ under the similarity equivalence in the space of square matrices $[1,6]$. From [1] we know that, for any $\mathbf{J}$, taking arbitrarily small parameters $\mathbf{q}$, we can obtain the matrix $\mathbf{J}+\mathbf{X}_{22}^{c}(\mathbf{q})$ having only simple eigenvalues. Then, taking arbitrarily small parameters in the block $\mathbf{X}_{21}^{c}(\mathbf{q})$, we can destroy the Jordan structure corresponding to every simple eigenvalue, leaving only one real generalized eigenvalue $\sigma$ or one pair of complex conjugate generalized eigenvalues $\sigma \pm i \omega$. Hence, an uncontrollable system of $\mathbf{J}_{\sigma}$ or $\mathbf{J}_{\sigma \pm i \omega}$ type can be found in any neighborhood of $\mathbf{q}=0$. The same holds for a generic family of matrix pairs $\alpha(\mathbf{p})$; i.e., a point $\mathbf{p}_{0} \in \mathcal{N}$ either lies on a surface represented by matrix pairs of $\mathbf{J}_{\sigma}$ or $\mathbf{J}_{\sigma \pm i \omega}$ types (regular points of $\mathcal{N}$ ) or belongs to a boundary of these surfaces.

Remark. Let us consider matrix pairs $\alpha$ with a fixed Jordan part $\mathbf{J}$ in the Brunovsky form. In the generic case, a Kronecker part $\mathbf{N}$ in the Brunovsky form of almost all matrix pairs $\alpha$ has maximal possible number of blocks $r$, and the sizes $k_{1}, \ldots, k_{r}$ of these blocks are different by no more than one, i.e., $k_{1}=\cdots=k_{r^{\prime}}=$ $k_{r^{\prime}+1}+1=\cdots=k_{r}+1[6]$. In this case $\mathbf{Y}_{11}^{c}=0$ and $\mathbf{Y}_{12}^{c}=0$ in versal deformation (3.5). Such a Kronecker part cannot be changed by a small perturbation of the parameters if we do not change the structure of the Jordan part. On the contrary, if the matrix pair has a different Kronecker part, then we can always find a nearby matrix pair with the generic Kronecker part, keeping the structure of the Jordan part [6].

4. Perturbation analysis of the uncontrollability set. Let us assume that we are given a point $\mathbf{p}_{0} \in \mathcal{N}$ corresponding to a pair $\alpha\left(\mathbf{p}_{0}\right)$ of $\mathbf{J}_{\sigma}$ or $\mathbf{J}_{\sigma \pm i \omega}$ type. From Theorem 3.1 we know the generic structure of $\mathcal{N}$ in the neighborhood of $\mathbf{p}_{0}$. Nevertheless, some symmetry or degeneracy of the family $\alpha(\mathbf{p})$ may cause the appearance of a nongeneric structure. For example, the pair

$$
\alpha(\mathbf{p})=\left(\left(\begin{array}{cc}
0 & 0 \\
0 & p_{1}+p_{2}+p_{3}
\end{array}\right),\left(\begin{array}{l}
1 \\
0
\end{array}\right)\right)
$$

is uncontrollable for all $\mathbf{p} \in \mathbb{R}^{3}$, and $\alpha(\mathbf{p})$ has $\mathbf{J}_{\sigma}$ type for any $\mathbf{p}$. Clearly, an arbitrarily small perturbation of the family can result in the generic structure of $\mathcal{N}$. For example, taking the $(2,1)$ th element of the first matrix to be $\epsilon p_{1}$ for an arbitrarily small $\epsilon>0$, the set $\mathcal{N}$ becomes the plane $p_{1}=0$ of codimension 1 , which is the generic case. Therefore, it would be useful to have a constructive criterion guaranteeing that the structure of $\mathcal{N}$ is generic for a given family $\alpha(\mathbf{p})$. For applications and numerical analysis of the uncontrollability set it is also important to have quantitative local information on $\mathcal{N}$, i.e., its tangent plane and perturbations of the generalized eigenvalues. The solution of these problems is given in the following theorems. 
Let $\mathbf{p}_{0}$ be a point of the uncontrollability set $\mathcal{N}$ for a family of matrix pairs $\alpha(\mathbf{p})$. Let $\gamma_{b}=(\mathbf{P}, \mathbf{Q}, \mathbf{R}) \in \mathcal{G}$ be a triple determining the feedback equivalence transformation of the pair $\alpha_{0}=\alpha\left(\mathbf{p}_{0}\right)$ to its Brunovsky canonical form $\alpha_{b}=\gamma_{b} \circ \alpha_{0}$.

For the pair $\alpha_{0}$ of $\mathbf{J}_{\sigma}$ type with the generalized eigenvalue $\sigma$, we define real vectors $\mathbf{f}_{i}=\left(f_{i}^{1}, \ldots, f_{i}^{k}\right), i=1, \ldots, m$, and $\mathbf{f}_{\sigma}=\left(f_{\sigma}^{1}, \ldots, f_{\sigma}^{k}\right)$ with the components

$$
\begin{aligned}
f_{i}^{j}= & \mathbf{P}^{-1}(n,:)\left[\frac{\partial \mathbf{A}}{\partial p_{j}} \sum_{s=1}^{k_{i}} \sigma^{s-1} \mathbf{P}\left(:, K_{i}+s\right)\right. \\
& \left.+\frac{\partial \mathbf{B}}{\partial p_{j}}\left(\sum_{s=1}^{k_{i}} \sigma^{s-1} \mathbf{R}\left(:, K_{i}+s\right)+\sigma^{k_{i}} \mathbf{Q}(:, i)\right)\right], i=1, \ldots, r ; \\
f_{i}^{j}= & \mathbf{P}^{-1}(n,:) \frac{\partial \mathbf{B}}{\partial p_{j}} \mathbf{Q}(:, i), \quad i=r+1, \ldots, m ; \\
f_{\sigma}^{j}= & \mathbf{P}^{-1}(n,:)\left[\frac{\partial \mathbf{A}}{\partial p_{j}} \mathbf{P}(:, n)+\frac{\partial \mathbf{B}}{\partial p_{j}} \mathbf{R}(:, n)\right]
\end{aligned}
$$

where $K_{1}=0, K_{i}=k_{1}+\cdots+k_{i-1} ; \mathbf{P}^{-1}(n,:), \mathbf{P}(:, i), \mathbf{Q}(:, i)$, and $\mathbf{R}(:, i)$ denote the $n$th row of $\mathbf{P}^{-1}$ and the $i$ th columns of $\mathbf{P}, \mathbf{Q}$, and $\mathbf{R}$, respectively.

THEOREM 4.1. Let us assume that $\alpha_{0}=\alpha\left(\mathbf{p}_{0}\right)$ is a matrix pair of $\mathbf{J}_{\sigma}$ type, and the vectors $\mathbf{f}_{1}, \ldots, \mathbf{f}_{m}$ are linearly independent. Then in the vicinity of $\mathbf{p}_{0}$ the uncontrollability set $\mathcal{N}$ is a smooth surface of codimension $m$ corresponding to matrix pairs of $\mathbf{J}_{\sigma}$ type. Its tangent plane at $\mathbf{p}_{0}$ is given by the equations

$$
\left(\mathbf{f}_{1}, \mathbf{p}-\mathbf{p}_{0}\right)=\cdots=\left(\mathbf{f}_{m}, \mathbf{p}-\mathbf{p}_{0}\right)=0,
$$

where $\left(\mathbf{f}_{i}, \mathbf{p}-\mathbf{p}_{0}\right)=\sum_{j=1}^{k} f_{i}^{j}\left(p_{j}-p_{0 j}\right)$ is a scalar product in $\mathbb{R}^{k}$. The generalized eigenvalue for $\mathbf{p} \in \mathcal{N}$ in the neighborhood of the point $\mathbf{p}_{0}$ is given by the expression

$$
\sigma+\left(\mathbf{f}_{\sigma}, \mathbf{p}-\mathbf{p}_{0}\right)+o\left(\left\|\mathbf{p}-\mathbf{p}_{0}\right\|\right) .
$$

For the pair $\alpha_{0}$ of $\mathbf{J}_{\sigma \pm i \omega}$ type with the generalized eigenvalues $\sigma \pm i \omega$, we define real vectors $\mathbf{f}_{s}=\left(f_{s}^{1}, \ldots, f_{s}^{k}\right), s=1, \ldots, 2 m, \mathbf{f}_{\sigma}=\left(f_{\sigma}^{1}, \ldots, f_{\sigma}^{k}\right)$, and $\mathbf{f}_{\omega}=\left(f_{\omega}^{1}, \ldots, f_{\omega}^{k}\right)$ with the components

$$
\begin{aligned}
& f_{2 s-1}^{j}+i f_{2 s}^{j}=\sum_{z=0}^{1} i^{1-z} \mathbf{P}^{-1}(n-z,:)\left[\frac{\partial \mathbf{A}}{\partial p_{j}} \sum_{v=1}^{k_{s}}(\sigma-i \omega)^{v-1} \mathbf{P}\left(:, K_{s}+v\right)\right. \\
& \left.+\frac{\partial \mathbf{B}}{\partial p_{j}}\left(\sum_{v=1}^{k_{s}}(\sigma-i \omega)^{v-1} \mathbf{R}\left(:, K_{s}+v\right)+(\sigma-i \omega)^{k_{s}} \mathbf{Q}(:, s)\right)\right], \quad s=1, \ldots, r
\end{aligned}
$$

$$
\begin{aligned}
& f_{2 s-1}^{j}+i f_{2 s}^{j}=\sum_{z=0}^{1} i^{1-z} \mathbf{P}^{-1}(n-z,:) \frac{\partial \mathbf{B}}{\partial p_{j}} \mathbf{Q}(:, s), \quad s=r+1, \ldots, m \\
& f_{\sigma}^{j}+i f_{\omega}^{j}=\frac{1}{2} \sum_{z=0}^{1} \sum_{v=0}^{1} i^{z-v} \mathbf{P}^{-1}(n-z,:)\left[\frac{\partial \mathbf{A}}{\partial p_{j}} \mathbf{P}(:, n-v)+\frac{\partial \mathbf{B}}{\partial p_{j}} \mathbf{R}(:, n-v)\right]
\end{aligned}
$$

where $i$ is the imaginary unit. 
TheOREM 4.2. Let us assume that $\alpha_{0}=\alpha\left(\mathbf{p}_{0}\right)$ is a matrix pair of $\mathbf{J}_{\sigma \pm i \omega}$ type, and the vectors $\mathbf{f}_{1}, \ldots, \mathbf{f}_{2 m}$ are linearly independent. Then in the vicinity of $\mathbf{p}_{0}$ the uncontrollability set $\mathcal{N}$ is a smooth surface of codimension $2 m$ corresponding to matrix pairs of $\mathbf{J}_{\sigma \pm i \omega}$ type. Its tangent plane at $\mathbf{p}_{0}$ is given by the equations

$$
\left(\mathbf{f}_{1}, \mathbf{p}-\mathbf{p}_{0}\right)=\cdots=\left(\mathbf{f}_{2 m}, \mathbf{p}-\mathbf{p}_{0}\right)=0 .
$$

The generalized eigenvalues for $\mathbf{p} \in \mathcal{N}$ in the neighborhood of $\mathbf{p}_{0}$ are given by the expression

$$
\sigma+\left(\mathbf{f}_{\sigma}, \mathbf{p}-\mathbf{p}_{0}\right) \pm i\left(\omega+\left(\mathbf{f}_{\omega}, \mathbf{p}-\mathbf{p}_{0}\right)\right)+o\left(\left\|\mathbf{p}-\mathbf{p}_{0}\right\|\right) .
$$

The important consequence of Theorems 4.1 and 4.2 is that to determine the local structure of the uncontrollability set we need only derivatives of the family $\alpha(\mathbf{p})$ with respect to the parameters at $\mathbf{p}_{0}$ and the triple $\gamma_{b}=(\mathbf{P}, \mathbf{Q}, \mathbf{R})$ transforming the pair $\alpha_{0}$ to the Brunovsky canonical form. The triple $\gamma_{b}$ can be found using the software developed in $[4,5]$, which provides the Kronecker canonical form of the matrix pencil $\left(\mathbf{A}_{0}, \mathbf{B}_{0}\right)-\lambda\left(\mathbf{I}_{n}, 0\right)$. The Brunovsky form can be obtained from the Kronecker canonical form by permutation of columns [9].

Example 4.1. Let us consider a three-parameter two-input system (2.1) with the matrices $\mathbf{A}$ and $\mathbf{B}$ given by the relations

$$
\mathbf{A}(\mathbf{p})=\left(\begin{array}{ccc}
-p_{3} & p_{1} p_{2} & p_{1} p_{2} \\
2-p_{3} & 3+p_{1} p_{2} & 1+p_{1} p_{2} \\
-2-2 p_{2}^{2} & p_{1}-1 & 1-p_{2}
\end{array}\right), \quad \mathbf{B}(\mathbf{p})=\left(\begin{array}{cc}
1 & -p_{2} \\
1 & 1-p_{2} \\
-1 & p_{2}-1
\end{array}\right) .
$$

The matrix pair $\alpha_{0}=\left(\mathbf{A}\left(\mathbf{p}_{0}\right), \mathbf{B}\left(\mathbf{p}_{0}\right)\right)$ at $\mathbf{p}_{0}=(0,0,0)$ is uncontrollable and has $\mathbf{J}_{\sigma}$ type. Its Brunovsky form consists of two $1 \times 1$ blocks $\mathbf{N}_{1}=\mathbf{N}_{2}=(0)$ in the Kronecker part $\left(r=2, k_{1}=k_{2}=1\right)$ and the Jordan part $\mathbf{J}=(\sigma)$ with the generalized eigenvalue $\sigma=2$. The transformation of $\alpha_{0}$ to the Brunovsky form is performed by the triple $\gamma_{b}=(\mathbf{P}, \mathbf{Q}, \mathbf{R})$ with the matrices

$$
\mathbf{P}=\left(\begin{array}{ccc}
1 & 0 & 0 \\
1 & 1 & 0 \\
-1 & -1 & 1
\end{array}\right), \mathbf{Q}=\left(\begin{array}{ll}
1 & 0 \\
0 & 1
\end{array}\right), \mathbf{R}=\left(\begin{array}{ccc}
0 & 0 & 0 \\
-4 & -2 & -1
\end{array}\right)
$$

Using formulae (4.2), we compute the vectors

$$
\mathbf{f}_{1}=(1,1,-1), \quad \mathbf{f}_{2}=(1,1,0), \quad \mathbf{f}_{\sigma}=(0,-1,0) .
$$

Since the vectors $\mathbf{f}_{1}$ and $\mathbf{f}_{2}$ are linearly independent, by Theorem 4.1, the uncontrollability set $\mathcal{N}$ is a smooth curve in the neighborhood of $\mathbf{p}_{0}=(0,0,0)$ with the tangent

$$
\left(\mathbf{f}_{1}, \mathbf{p}-\mathbf{p}_{0}\right)=p_{1}+p_{2}-p_{3}=0, \quad\left(\mathbf{f}_{2}, \mathbf{p}-\mathbf{p}_{0}\right)=p_{1}+p_{2}=0 .
$$

The generalized eigenvalue of the matrix pair $\alpha(\mathbf{p})$ for $\mathbf{p} \in \mathcal{N}$ is approximated by

$$
\sigma+\left(\mathbf{f}_{\sigma}, \mathbf{p}-\mathbf{p}_{0}\right)+o\left(\left\|\mathbf{p}-\mathbf{p}_{0}\right\|\right)=2-p_{2}+o(\|\mathbf{p}\|) .
$$

It is straightforward to check that $\alpha(\mathbf{p})=\gamma(\mathbf{p}) \circ \beta(\mathbf{q}(\mathbf{p}))$, where

$$
\beta(\mathbf{q})=\left(\left(\begin{array}{ccc}
0 & 0 & 0 \\
0 & 0 & 0 \\
q_{1} & q_{2} & 2+q_{3}
\end{array}\right),\left(\begin{array}{cc}
1 & 0 \\
0 & 1 \\
0 & 0
\end{array}\right)\right), \begin{aligned}
& q_{1}(\mathbf{p})=p_{1}+p_{2}-p_{3}-2 p_{2}^{2} \\
& q_{2}(\mathbf{p})=p_{1}+p_{2} \\
& q_{3}(\mathbf{p})=-p_{2}+p_{1} p_{2}
\end{aligned}
$$




$$
\gamma(\mathbf{p})=\left(\left(\begin{array}{ccc}
1 & 0 & 0 \\
-1 & 1 & 0 \\
0 & 1 & 1
\end{array}\right),\left(\begin{array}{cc}
1 & -p_{2} \\
0 & 1
\end{array}\right),\left(\begin{array}{ccc}
-p_{3} & p_{1} p_{2} & p_{1} p_{2} \\
2 & 3 & 1
\end{array}\right)\right)
$$

Hence, we find exact expressions for the uncontrollability set and the generalized eigenvalue in the form

$$
\begin{gathered}
\mathcal{N}=\left\{\mathbf{p} \in \mathbb{R}^{3} \mid p_{1}+p_{2}-p_{3}-2 p_{2}^{2}=0, p_{1}+p_{2}=0\right\} \\
\sigma(\mathbf{p})=2-p_{2}+p_{1} p_{2} .
\end{gathered}
$$

This agrees with the results obtained by Theorem 4.1 .

Proof of Theorem 4.1. Let us consider a family of matrix pairs $\widetilde{\alpha}(\mathbf{p})=\gamma_{b} \circ \alpha(\mathbf{p})$. This family is a deformation of the Brunovsky canonical form $\widetilde{\alpha}\left(\mathbf{p}_{0}\right)=\alpha_{b}$. By Theorem 2.1 , the family $\widetilde{\alpha}(\mathbf{p})$ can be represented in the form $\widetilde{\alpha}(\mathbf{p})=\widetilde{\gamma}(\mathbf{p}) \circ \beta(\mathbf{q}(\mathbf{p}))$, where $\beta(\mathbf{q})$ is the versal deformation of $\alpha_{b}$ having the form (3.5), (3.6). Since the controllability property is invariant under the feedback equivalence transformation, the uncontrollability sets for the families $\alpha(\mathbf{p})$ and $\beta(\mathbf{q}(\mathbf{p}))$ coincide in the neighborhood of $\mathbf{p}_{0}$ and, according to (3.7), have the form

$$
q_{1}(\mathbf{p})=\cdots=q_{m}(\mathbf{p})=0,
$$

where $q_{1}\left(\mathbf{p}_{0}\right)=\cdots=q_{m}\left(\mathbf{p}_{0}\right)=0$ by construction. Derivatives of the functions $q_{i}(\mathbf{p})$ at $\mathbf{p}_{0}$ are given by expression (2.18) of Theorem 2.1, where we take $\widetilde{\alpha}(\mathbf{p})$ and $\alpha_{b}$ instead of $\alpha(\mathbf{p})$ and $\alpha_{0}$, respectively. Formula (2.18) requires the basis $\left\{\alpha_{1}^{n}, \ldots, \alpha_{\ell}^{n}\right\}$ of the subspace $\left(T_{\alpha_{b}} \mathcal{O}\left(\alpha_{b}\right)\right)^{\perp}$. This subspace for a matrix pair in the Brunovsky canonical form was found explicitly in [6]. It is convenient to represent the basis of $\left(T_{\alpha_{b}} \mathcal{O}\left(\alpha_{b}\right)\right)^{\perp}$ in the form of the family $\alpha_{1}^{n} q_{1}+\cdots+\alpha_{\ell}^{n} q_{\ell}$, which, for the matrix pair $\alpha_{b}$ under consideration, takes the form

$$
\left(\left(\begin{array}{cc}
\mathbf{X}_{11}^{n} & 0 \\
\mathbf{X}_{21}^{n} & \mathbf{X}_{22}^{n}
\end{array}\right),\left(\begin{array}{cc}
\mathbf{Y}_{11}^{n} & \mathbf{Y}_{12}^{n} \\
\mathbf{Y}_{21}^{n} & \mathbf{Y}_{22}^{n}
\end{array}\right)\right)
$$

where

$$
\begin{aligned}
& \mathbf{X}_{21}^{n}=\left(\mathbf{L}_{1}^{n}\left(q_{1}\right), \ldots, \mathbf{L}_{r}^{n}\left(q_{r}\right)\right), \quad \mathbf{X}_{22}^{n}=\left(q_{m+1}\right), \\
& \mathbf{Y}_{21}^{n}=\left(\sigma^{k_{1}} q_{1}, \ldots, \sigma^{k_{r}} q_{r}\right), \quad \mathbf{Y}_{22}^{n}=\left(q_{r+1}, \ldots, q_{m}\right) ;
\end{aligned}
$$

$\mathbf{L}_{i}^{n}\left(q_{i}\right)=\left(q_{i}, \sigma q_{i}, \ldots, \sigma^{k_{i}-1} q_{i}\right)$ is a $1 \times k_{i}$ matrix. The blocks $\mathbf{X}_{11}^{n}(\mathbf{q}), \mathbf{Y}_{11}^{n}(\mathbf{q})$, and $\mathbf{Y}_{12}^{n}(\mathbf{q})$ depend on $q_{m+2}, \ldots, q_{\ell}$. Comparing this basis with the basis $\left\{\alpha_{1}^{c}, \ldots, \alpha_{\ell}^{c}\right\}$ defined by (3.5), (3.6), we see that $z_{i j}=\left\langle\alpha_{j}^{c}, \alpha_{i}^{n}\right\rangle_{1}=\delta_{i j}$ if $i \leq m+1$ or $j \leq m+1$, where $\delta_{i j}$ is the Kronecker delta. Hence, using (2.18), we find the derivatives of $q_{1}(\mathbf{p}), \ldots, q_{m+1}(\mathbf{p})$ at $\mathbf{p}_{0}$ in the form

$$
\frac{\partial q_{i}}{\partial p_{j}}=\left\langle\frac{\partial \widetilde{\alpha}}{\partial p_{j}}, \alpha_{i}^{n}\right\rangle_{1}, \quad i=1, \ldots, m+1 .
$$

Using $\widetilde{\alpha}(\mathbf{p})$ in its original form $\gamma_{b} \circ \alpha(\mathbf{p})=\left(\mathbf{P}^{-1}(\mathbf{A}(\mathbf{p}) \mathbf{P}+\mathbf{B}(\mathbf{p}) \mathbf{R}), \mathbf{P}^{-1} \mathbf{B}(\mathbf{p}) \mathbf{Q}\right)$ and the explicit form of $\alpha_{i}^{n}$, we find

$$
\frac{\partial q_{i}}{\partial p_{j}}=f_{i}^{j}, \quad i=1, \ldots, m ; \quad \frac{\partial q_{m+1}}{\partial p_{j}}=f_{\sigma}^{j}
$$


with $f_{i}^{j}$ and $f_{\sigma}^{j}$ defined in (4.2). Therefore, $\mathbf{f}_{1}, \ldots, \mathbf{f}_{m}$, and $\mathbf{f}_{\sigma}$ are the gradient vectors of the functions $q_{1}(\mathbf{p}), \ldots, q_{m+1}(\mathbf{p})$ at $\mathbf{p}_{0}$ :

$$
\nabla q_{i}=\mathbf{f}_{i}, \quad i=1, \ldots, m ; \quad \nabla q_{m+1}=\mathbf{f}_{\sigma} ; \quad \nabla=\left(\frac{\partial}{\partial p_{1}}, \ldots, \frac{\partial}{\partial p_{k}}\right) .
$$

If the gradient vectors $\nabla q_{1}, \ldots, \nabla q_{m}$ are linearly independent, then, by the implicit function theorem, the set $\mathcal{N}$ determined by (4.15) is a smooth surface of codimension $m$ with the tangent plane (4.3). If $q_{1}(\mathbf{p})=\cdots=q_{m}(\mathbf{p})=0$, then we see from (3.5), (3.6) that $\beta(\mathbf{q})$ and, hence, $\alpha(\mathbf{p})$ is a matrix pair of $\mathbf{J}_{\sigma}$ type with the generalized eigenvalue $\sigma+q_{m+1}(\mathbf{p})=\sigma+\left(\mathbf{f}_{\sigma}, \mathbf{p}-\mathbf{p}_{0}\right)+o\left(\left\|\mathbf{p}-\mathbf{p}_{0}\right\|\right)$.

Proof of Theorem 4.2. The case when the pair $\alpha_{0}$ has $\mathbf{J}_{\sigma \pm i \omega}$ type is studied analogously. In this case the versal deformation $\beta(\mathbf{q})$ takes the form (3.5), (3.8), (3.9), and the basis $\left\{\alpha_{1}^{n}, \ldots, \alpha_{\ell}^{n}\right\}$ is represented by family (4.16) with

$$
\begin{aligned}
& \mathbf{X}_{21}^{n}=\left[\mathbf{L}_{1}^{n}\left(q_{1}, q_{2}\right), \ldots, \mathbf{L}_{r}^{n}\left(q_{2 r-1}, q_{2 r}\right)\right], \\
& \mathbf{X}_{22}^{n}=\left(\begin{array}{cc}
q_{2 m+1} / 2 & q_{2 m+2} / 2 \\
-q_{2 m+2} / 2 & q_{2 m+1} / 2
\end{array}\right), \\
& \mathbf{Y}_{21}^{n}=\left(\begin{array}{ccc}
\operatorname{Re}\left(q_{1}+i q_{2}\right)(\sigma+i \omega)^{k_{1}} & \ldots & \operatorname{Re}\left(q_{2 r-1}+i q_{2 r}\right)(\sigma+i \omega)^{k_{r}} \\
\operatorname{Im}\left(q_{1}+i q_{2}\right)(\sigma+i \omega)^{k_{1}} & \ldots & \operatorname{Im}\left(q_{2 r-1}+i q_{2 r}\right)(\sigma+i \omega)^{k_{r}}
\end{array}\right) \\
& \mathbf{Y}_{22}^{n}=\left(\begin{array}{ccc}
q_{2 r+1} & \cdots & q_{2 m-1} \\
q_{2 r+2} & \cdots & q_{2 m}
\end{array}\right),
\end{aligned}
$$

where $\mathbf{L}_{s}^{n}\left(q_{2 s-1}, q_{2 s}\right)$ is a $2 \times k_{s}$ matrix of the form

$$
\mathbf{L}_{s}^{n}=\left(\begin{array}{cccc}
q_{2 s-1} & \sigma q_{2 s-1}-\omega q_{2 s} & \cdots & \operatorname{Re}\left(q_{2 s-1}+i q_{2 s}\right)(\sigma+i \omega)^{k_{s}-1} \\
q_{2 s} & \omega q_{2 s-1}+\sigma q_{2 s} & \cdots & \operatorname{Im}\left(q_{2 s-1}+i q_{2 s}\right)(\sigma+i \omega)^{k_{s}-1}
\end{array}\right),
$$

and the blocks $\mathbf{X}_{11}^{n}, \mathbf{Y}_{11}^{n}$, and $\mathbf{Y}_{12}^{n}$ depend on $q_{2 m+3}, \ldots, q_{\ell}$. The uncontrollability set is given by the equations

$$
q_{1}(\mathbf{p})=\cdots=q_{2 m}(\mathbf{p})=0,
$$

where the gradients $\nabla q_{1}, \ldots, \nabla q_{2 m+2}$ are equal to the vectors $\mathbf{f}_{1}, \ldots, \mathbf{f}_{2 m}, \mathbf{f}_{\sigma}, \mathbf{f}_{\omega}$ defined in (4.5)-(4.7). The generalized eigenvalues on the surface (4.23) are determined by the expression

$$
\begin{aligned}
\sigma+q_{2 m+1}(\mathbf{p}) \pm i\left(\omega+q_{2 m+2}(\mathbf{p})\right)= & \sigma+\left(\mathbf{f}_{\sigma}, \mathbf{p}-\mathbf{p}_{0}\right) \pm i\left(\omega+\left(\mathbf{f}_{\omega}, \mathbf{p}-\mathbf{p}_{0}\right)\right) \\
& +o\left(\left\|\mathbf{p}-\mathbf{p}_{0}\right\|\right) .
\end{aligned}
$$

5. Numerical construction of the uncontrollability set. Perturbation analysis developed in the previous section can be applied to numerical construction of the uncontrollability set by continuation if one point of this set is known.

Let us illustrate the implementation of this procedure for a specific case of a threeparameter system with one-input variable $(k=3$ and $m=1)$; dimension of the state space is arbitrary. Let us assume that we are given a point $\mathbf{p}_{0} \in \mathcal{N}$ corresponding to 
a matrix pair $\alpha\left(\mathbf{p}_{0}\right)$ of $\mathbf{J}_{\sigma \pm i \omega}$ type. By Theorem 3.1, in the generic case the set $\mathcal{N}$ is a smooth curve in a vicinity of the point $\mathbf{p}_{0}$. Let us introduce the length parameter $y$ along the curve $\mathcal{N}$. Then the curve $\mathcal{N}$ is given by a smooth function $\mathbf{p}(y)$ such that $\|d \mathbf{p} / d y\|=1$. By Theorem 4.2 , the vectors $\mathbf{f}_{1}, \mathbf{f}_{2} \in \mathbb{R}^{3}$ evaluated by expressions (4.5), (4.6) at $\mathbf{p}(y)$ are normal vectors to the curve $\mathcal{N}$ at $\mathbf{p}(y)$. The vector product $\mathbf{f}_{1} \times \mathbf{f}_{2}$ is a tangent vector to $\mathcal{N}$. Hence, we find

$$
\frac{d \mathbf{p}}{d y}=\mathbf{g}, \quad \mathbf{g}=\frac{\mathbf{f}_{1} \times \mathbf{f}_{2}}{\left\|\mathbf{f}_{1} \times \mathbf{f}_{2}\right\|} .
$$

Using expressions (4.9) and (5.1), we find derivatives of the functions $\sigma(y)$ and $\omega(y)$, which determine the generalized eigenvalues $\sigma(y) \pm i \omega(y)$ of the matrix pair $\alpha(\mathbf{p}(y))$, as follows:

$$
\frac{d \sigma}{d y}=\left(\mathbf{f}_{\sigma}, \mathbf{g}\right), \quad \frac{d \omega}{d y}=\left(\mathbf{f}_{\omega}, \mathbf{g}\right) .
$$

Equations (5.1) and (5.2) represent a system of ordinary differential equations with respect to $\mathbf{p}, \sigma$, and $\omega$. Initial conditions at $y=0$ are given by

$$
\mathbf{p}(0)=\mathbf{p}_{0}, \quad \sigma(0)=\sigma_{0}, \quad \omega(0)=\omega_{0},
$$

where $\sigma_{0} \pm i \omega_{0}$ are generalized eigenvalues of the matrix pair $\alpha\left(\mathbf{p}_{0}\right)$ at the given point $\mathbf{p}_{0} \in \mathcal{N}$. Integrating this system forwards and backwards, we find a regular part of the uncontrollability set of $\mathbf{J}_{\sigma \pm i \omega}$ type. Since we use the local information on the uncontrollability set, there is no guarantee that we have found the whole set $\mathcal{N}$ in the parameter space. Nevertheless, we obtain a finite piece of the uncontrollability set containing the given point $\mathbf{p}_{0}$. The integration can be continued until we reach a physical boundary of the parameter space or arrive at a singularity (boundary of the regular part of $\mathcal{N}$ ). A singularity causes numerical instability of the integration procedure and can be recognized by the appearance of a matrix pair with a different Jordan structure.

To determine right-hand sides of system (5.1), (5.2), we need to find the transformation to the Brunovsky canonical form

$$
\alpha_{b}(y)=\gamma_{b}(y) \circ \alpha(\mathbf{p}(y))
$$

at each $y$. This can be done using the software $[4,5]$ providing the Kronecker canonical form of the matrix pencil $(\mathbf{A}(\mathbf{p}(y)), \mathbf{B}(\mathbf{p}(y)))-\lambda\left(\mathbf{I}_{n}, 0\right)$. Then permutation of columns provides the Brunovsky canonical form [9]. In these calculations, the information on the generalized eigenvalues $\sigma(y) \pm i \omega(y)$ can be used.

Alternatively, we can calculate $\alpha_{b}(y)$ and $\gamma_{b}(y)$ by taking advantage of the remark in section 3. In the generic case, the Brunovsky form $\alpha_{b}(y)$ has one $(n-2) \times(n-2)$ block $\mathbf{N}_{1}$ in the Kronecker part:

$$
\alpha_{b}(y)=\left(\left(\begin{array}{cc}
\mathbf{N}_{1} & 0 \\
0 & \mathbf{J}_{\sigma \pm i \omega}
\end{array}\right),\left(\begin{array}{c}
\mathbf{E}_{1} \\
0
\end{array}\right)\right) .
$$

Taking the derivative of (5.4) with respect to $y$, we find

$$
\frac{d \alpha_{b}}{d y}=d f_{\alpha_{b}}\left(\gamma_{b}^{-1} \frac{d \gamma_{b}}{d y}\right)+\gamma_{b} \circ \frac{d \alpha(\mathbf{p}(y))}{d y},
$$


where $d f_{\alpha_{b}}(\xi)$ is the differential of the function $f_{\alpha_{b}}(\gamma)$ at the unit element $e$ defined by expression (2.12). Introducing the matrix triple $\xi=\gamma_{b}^{-1} d \gamma_{b} / d y$ and using expression (5.1), we find a linear algebraic equation

$$
d f_{\alpha_{b}}(\xi)=\frac{d \alpha_{b}}{d y}-\gamma_{b} \circ\left(\sum_{i=1}^{k} \frac{\partial \alpha}{\partial p_{i}} g_{i}\right) .
$$

Then the derivative of the function $\gamma_{b}(y)$ is given by the relation

$$
\frac{d \gamma_{b}}{d y}=\gamma_{b}(y) \xi
$$

Equation (5.7) does not determine the triple $\xi$ uniquely (transformation to the Brunovsky form is not unique). It is convenient to choose a particular solution satisfying the condition

$$
\xi \in\left(\operatorname{Ker} d f_{\alpha_{b}}\right)^{\perp},
$$

which determines a unique $\xi$. A numerical method for finding this solution is given in the appendix.

Differential equation (5.8) can be integrated together with system (5.1), (5.2). As a result, we find the Brunovsky canonical form $\alpha_{b}(y)$ and the feedback equivalence transformation $\gamma_{b}(y)$ at each point of the uncontrollability set represented by the curve $\mathbf{p}(y)$. The initial conditions are given by

$$
\gamma_{b}(0)=\gamma_{b}^{0},
$$

where $\gamma_{b}^{0}$ transforms the matrix pair $\alpha\left(\mathbf{p}_{0}\right)$ to the Brunovsky form. Note that matrices of the triple $\gamma_{b}(y)=\left(\mathbf{P}_{b}(y), \mathbf{Q}_{b}(y), \mathbf{R}_{b}(y)\right)$ may become ill-conditioned when approaching a singularity of $\mathcal{N}$. To control the accuracy of calculations and to detect a singularity it is convenient to use the norm $\left\|\alpha_{b}(y)-\gamma_{b}(y) \circ \alpha(\mathbf{p}(y))\right\|$, which describes the error in equality (5.4).

Similarly, we can calculate a regular part of the uncontrollability set, corresponding to matrix pairs of $\mathbf{J}_{\sigma}$ type, which is a smooth surface for a three-parameter oneinput dynamical system. Recall that, by Theorem 3.1, the surfaces corresponding to matrix pairs of $\mathbf{J}_{\sigma}$ and $\mathbf{J}_{\sigma \pm i \omega}$ types, together with their boundaries, form the whole uncontrollability set of a generic multi-input linear dynamical system.

Example 5.1. Let us consider a mechanical system shown in Figure 5.1. The system consists of a thin uniform platform of mass $m$ and length $2 l$ supported at both ends by springs having elastic coefficients $k_{1}, k_{2}$ and viscous damping coefficients $d_{1}$, $d_{2}$. There is a vertical force $F$ applied to the platform at the distance $\xi l$ from the middle. As generalized coordinates, we take a vertical coordinate $z$ of the center of the platform and an angle $\varphi$ between the platform and horizontal axis. The equilibrium of the system for zero external force $F=0$ is assumed to be $z=0, \varphi=0$.

Equations of motion of the system linearized near the equilibrium take the form

$$
\begin{aligned}
& m \ddot{z}+d_{1}(\dot{z}+l \dot{\varphi})+d_{2}(\dot{z}-l \dot{\varphi})+k_{1}(z+l \varphi)+k_{2}(z-l \varphi)=F, \\
& I_{m} \ddot{\varphi}+d_{1} l(\dot{z}+l \dot{\varphi})-d_{2} l(\dot{z}-l \dot{\varphi})+k_{1} l(z+l \varphi)-k_{2} l(z-l \varphi)=-\xi l F,
\end{aligned}
$$

where $I_{m}=m l^{2} / 3$ is the moment of inertia of the platform with respect to the center of mass; the dot denotes differentiation with respect to time $t$. If $F=0$, then the 


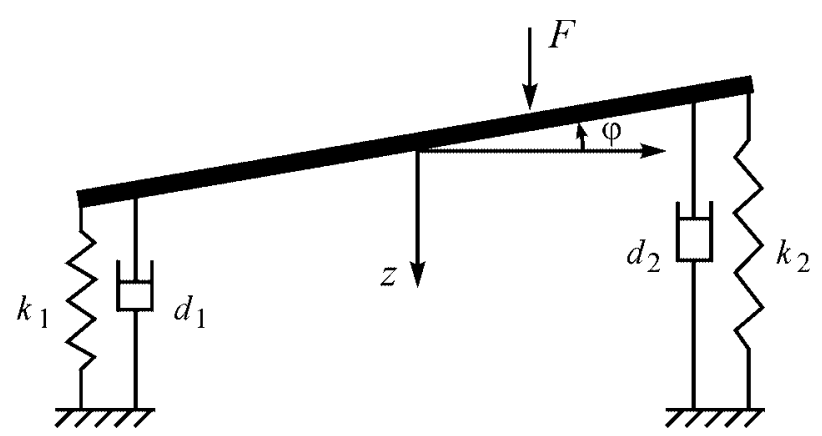

FIG. 5.1. Mechanical system controlled by a vertical force.

system oscillates with a decaying amplitude and comes to the equilibrium in infinite time $t \longrightarrow+\infty$. Let us consider the force $F$ as a control parameter. If the system is controllable, then it can be damped (put into the equilibrium) in finite time. This task becomes difficult or impossible if the system is close to the uncontrollable system.

Let us introduce nondimensional variables

$$
\begin{aligned}
& \tau=t / \alpha, \quad f_{1}=\frac{\left(d_{1}+d_{2}\right) \alpha}{m}, \quad f_{2}=\frac{\left(d_{1}-d_{2}\right) \alpha}{m}, \\
& c_{1}=\frac{\left(k_{1}+k_{2}\right) \alpha^{2}}{m}, \quad c_{2}=\frac{\left(k_{1}-k_{2}\right) \alpha^{2}}{m}, \quad u=\frac{\alpha^{2}}{m l} F,
\end{aligned}
$$

where $\alpha$ is a time scale, and choose a state vector $\mathbf{x} \in \mathbb{R}^{4}$ in the form

$$
x_{1}=\frac{z}{l}, \quad x_{2}=\varphi, \quad x_{3}=\frac{\alpha \dot{z}}{l}, \quad x_{4}=\alpha \dot{\varphi}
$$

Then system (5.11) can be written in the form $\dot{\mathbf{x}}=\mathbf{A} \mathbf{x}+\mathbf{B} u$, where derivative is taken with respect to nondimensional time $\tau$, and the matrices $\mathbf{A}$ and $\mathbf{B}$ are

$$
\mathbf{A}=\left(\begin{array}{cccc}
0 & 0 & 1 & 0 \\
0 & 0 & 0 & 1 \\
-c_{1} & -c_{2} & -f_{1} & -f_{2} \\
-3 c_{2} & -3 c_{1} & -3 f_{2} & -3 f_{1}
\end{array}\right), \quad \mathbf{B}=\left(\begin{array}{c}
0 \\
0 \\
1 \\
-3 \xi
\end{array}\right)
$$

Let us fix the parameters $c_{1}=25 / 12$ and $f_{1}=1$, characterizing the joint stiffness and damping of supports, and consider the parameter vector $\mathbf{p}=\left(c_{2}, f_{2}, \xi\right)$. Let us consider the point $\mathbf{p}_{0}=(0,0,0)$ corresponding to equal supports and the force applied at the center of the platform. The matrix pair $\alpha\left(\mathbf{p}_{0}\right)$ is uncontrollable and has the Brunovsky canonical form

$$
\mathbf{A}_{b}=\left(\begin{array}{cccc}
0 & 1 & 0 & 0 \\
0 & 0 & 0 & 0 \\
0 & 0 & \sigma_{0} & \omega_{0} \\
0 & 0 & -\omega_{0} & \sigma_{0}
\end{array}\right), \quad \mathbf{B}_{b}=\left(\begin{array}{c}
0 \\
1 \\
0 \\
0
\end{array}\right)
$$

with the uncontrollable modes $\sigma_{0} \pm i \omega_{0}=-1.5 \pm i 2$. The triple $\gamma_{b}^{0}=\left(\mathbf{P}_{b}, \mathbf{Q}_{b}, \mathbf{R}_{b}\right)$ 
a

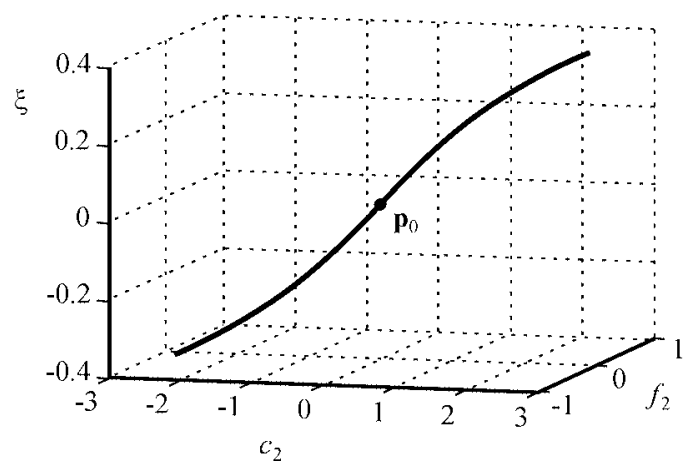

b

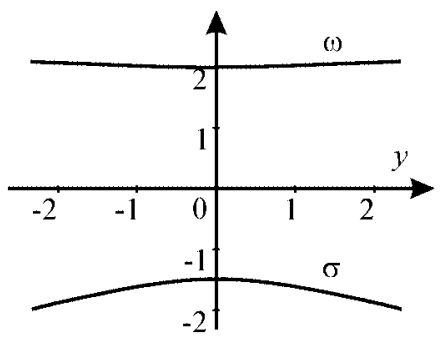

FIG. 5.2. (a) Uncontrollability set in the parameter space; (b) uncontrollable modes $\sigma(y) \pm i \omega(y)$.

transforming $\alpha\left(\mathbf{p}_{0}\right)$ to the Brunovsky form is

$$
\mathbf{P}_{b}=\left(\begin{array}{cccc}
1 & 0 & 0 & 0 \\
0 & 0 & 1 & 0 \\
0 & 1 & 0 & 0 \\
0 & 0 & -1.5 & 2
\end{array}\right), \quad \mathbf{Q}_{b}=(1), \quad \mathbf{R}_{b}=\left(\begin{array}{cccc}
25 / 12 & 1 & 0 & 0
\end{array}\right) .
$$

The point $\mathbf{p}_{0}$ belongs to the uncontrollability set and has $\mathbf{J}_{\sigma \pm i \omega}$ type. Integrating equations (5.1), (5.2), (5.8) with initial conditions (5.3), (5.10) numerically, we find the uncontrollability set $\mathcal{N}$ in the physical range of parameters $-c_{1}<c_{2}<c_{1}$ and $-f_{1}<f_{2}<f_{1}$ corresponding to positive characteristics of the supports. The result is shown in Figure 5.2(a), where the set $\mathcal{N}$ is represented by a solid line. Figure 5.2(b) shows graphs of the real and imaginary parts of the uncontrollable modes $\sigma(y) \pm i \omega(y)$. The maximal error $\left\|\alpha_{b}(y)-\gamma_{b}(y) \circ \alpha(\mathbf{p}(y))\right\|$ along the curve $\mathcal{N}$ is about $4 \cdot 10^{-7}$, which is less than the accuracy of the ordinary differential equation solver (the calculations were carried out in MATLAB using the standard ode45 solver).

Using the computed data, it can be shown that one mode of free vibrations of the system with the parameter vector $\mathbf{p}(y)=\left(c_{2}(y), f_{2}(y), \xi(y)\right)$ has a node at the distance $\xi(y) l$ from the center of the platform; i.e., this mode represents rotation of the platform around a point. The force $F$ applied at this point has no influence on the rotational mode, which leads to uncontrollability of the system. From Figure 5.2(a) we see that uncontrollability occurs when one of the supports has smaller stiffness and damping coefficients, and the force is applied at the side of a softer support. The obtained results are useful for the design of the system to avoid uncontrollability effects.

6. Singularities of the uncontrollability set. In this section we consider points of the uncontrollability set corresponding to matrix pairs whose types are different from $\mathbf{J}_{\sigma}$ and $\mathbf{J}_{\sigma \pm i \omega}$. By Theorem 3.1, these points belong to a boundary of the regular part of $\mathcal{N}$, represented by matrix pairs of $\mathbf{J}_{\sigma}$ and $\mathbf{J}_{\sigma \pm i \omega}$ types, and determine singularities (nonsmooth points) of the uncontrollability set.

In order to understand the role of singular points in the structure of the uncontrollability set, let us consider a specific case when the Jordan part of the Brunovsky 
canonical form $\alpha_{b}$ of a pair $\alpha(\mathbf{p}), \mathbf{p} \in \mathcal{N}$, is a $2 \times 2$ Jordan block

$$
\mathbf{J}_{\sigma^{2}}=\left(\begin{array}{cc}
\sigma & 1 \\
0 & \sigma
\end{array}\right)
$$

with a double real generalized eigenvalue $\sigma$. In the generic case, points $\mathbf{p} \in \mathcal{N}$ corresponding to matrix pairs of this type form a smooth surface of codimension $2 m+1$ in the parameter space [6]. If the number of parameters is less than $2 m+1$, then matrix pairs of $\mathbf{J}_{\sigma^{2}}$ type do not appear in generic families $\alpha(\mathbf{p})$.

In the case under consideration, a versal deformation of the pair $\alpha_{b}$ has the form (3.5), where [6]

$$
\begin{gathered}
\mathbf{X}_{21}^{c}=\left(\mathbf{L}_{1}^{c}\left(q_{1}, q_{2}\right), \ldots, \mathbf{L}_{r}^{c}\left(q_{2 r-1}, q_{2 r}\right)\right), \\
\mathbf{Y}_{22}^{c}=\left(\begin{array}{ccc}
q_{2 r+1} & \cdots & q_{2 m-1} \\
q_{2 r+2} & \cdots & q_{2 m}
\end{array}\right), \quad \mathbf{X}_{22}^{c}=\left(\begin{array}{cc}
q_{2 m+2} & 0 \\
q_{2 m+1} & q_{2 m+2}
\end{array}\right),
\end{gathered}
$$

and $\mathbf{L}_{i}^{c}\left(q_{2 i-1}, q_{2 i}\right)$ is a $2 \times k_{i}$ matrix of the form

$$
\mathbf{L}_{i}^{c}\left(q_{2 i-1}, q_{2 i}\right)=\left(\begin{array}{cccc}
q_{2 i-1} & 0 & \cdots & 0 \\
q_{2 i} & 0 & \cdots & 0
\end{array}\right) ;
$$

the blocks $\mathbf{Y}_{11}^{c}$ and $\mathbf{Y}_{12}^{c}$ depend on $q_{2 m+3}, \ldots, q_{\ell}$. Using controllability condition (2.2), we find that the uncontrollability set of the versal deformation in the neighborhood of $\mathbf{q}=0$ is determined by the equations

$$
q_{2 i-1}^{2} q_{2 m+1}-q_{2 i}^{2}=0, \quad i=1, \ldots, m .
$$

Every equation in (6.4) determines in the space $\left(q_{2 i-1}, q_{2 i}, q_{2 m+1}\right)$ a surface shown in Figure 3.1 and discussed in Example 3.1. Hence, the regular part of the uncontrollability set consists of one smooth surface of $\mathbf{J}_{\sigma \pm i \omega}$ type and codimension $2 m$, determined by the equations

$$
q_{i}=0, \quad i=1, \ldots, 2 m, \quad q_{2 m+1}<0,
$$

and smooth surfaces of $\mathbf{J}_{\sigma}$ type determined by the equations

$$
q_{2 i-1}^{2} q_{2 m+1}-q_{2 i}^{2}=0, \quad q_{2 i-1} \neq 0, \quad i=1, \ldots, m, \quad q_{2 m+1} \geq 0 .
$$

There are $2^{m}$ separate surfaces of $\mathbf{J}_{\sigma}$ type corresponding to different combinations of signs of the parameters $q_{2 i-1}, i=1, \ldots, m$, in (6.6). The singular part of $\mathcal{N}$ is a boundary of the regular part. It consists of several smooth surfaces, which are parts of the set (6.4) with the additional condition

$$
q_{2 j-1}=q_{2 j}=0, \quad q_{2 m+1} \geq 0
$$

for some $j \in\{1, \ldots, m\}$.

The structure of the uncontrollability set for a generic family $\alpha(\mathbf{p})$ in the neighborhood of a point $\mathbf{p}_{0} \in \mathcal{N}$ of $\mathbf{J}_{\sigma^{2}}$ type is the same as for the versal deformation. These sets are related by a smooth change of parameters $\mathbf{q}=\mathbf{q}(\mathbf{p})$. Analogously to the method of Theorem 4.1, we can use formulae of Theorem 2.1 to calculate the gradients $\nabla q_{i}$ of the functions $q_{i}(\mathbf{p})$ at the singular point $\mathbf{p}_{0}$. Then expressions (6.4)(6.7), where $q_{i}(\mathbf{p})$ is substituted by its linear approximation $\left(\nabla q_{i}, \mathbf{p}-\mathbf{p}_{0}\right)$, provide first-order approximations of the regular and singular parts of $\mathcal{N}$. 
Singular points lead to a more rich and complicated structure of the uncontrollability set. This affects the behavior of the underlying dynamical system and causes numerical difficulties in the analysis of $\mathcal{N}$. The information on the local form of $\mathcal{N}$ at its regular or singular point is useful for the analysis and construction of the uncontrollability set. In particular, this information allows choosing the locally optimal change of design parameters in order to get a controllable system.

7. Conclusion. In this paper, fundamental properties of the uncontrollability set for a multi-input linear dynamical system dependent on parameters are investigated. It is shown that the uncontrollability set has a regular part, which consists of smooth surfaces corresponding to one real uncontrollable mode or a complex conjugate pair of uncontrollable modes. Explicit formulae for local quantitative analysis of the uncontrollability set and perturbation of the uncontrollable modes are derived and used for numerical construction of the uncontrollability set in the parameter space.

A constructive method for qualitative and quantitative analysis of the uncontrollability set based on the versal deformation theory is developed. The idea of regularization of the parameter space by transformation to a versal deformation, proposed in the paper, provides a powerful tool of multiparameter perturbation theory for control systems.

Using the duality theorem [3, 13], all the results of this paper can be applied to the analysis of an unobservability set for a multioutput linear dynamical system dependent on parameters.

Appendix. Let us consider the linear algebraic equation with respect to $\xi \in T_{e} \mathcal{G}$

$$
d f_{\alpha}(\xi)=\alpha^{\prime}, \quad \xi \in\left(\operatorname{Ker} d f_{\alpha}\right)^{\perp},
$$

assuming that a solution exists, i.e., $\alpha^{\prime} \in \operatorname{Im} d f_{\alpha}$. We denote by $\operatorname{vec}(\mathbf{A})$ a column vector, which is an ordered stack of columns of $\mathbf{A}$ from left to right (its dimension is equal to the number of elements of $\mathbf{A})$. Analogously, we introduce the vectorization of the matrix pairs $\alpha=(\mathbf{A}, \mathbf{B}), \alpha^{\prime}=\left(\mathbf{A}^{\prime}, \mathbf{B}^{\prime}\right)$, and matrix triple $\xi=(\mathbf{U}, \mathbf{V}, \mathbf{W})$ as follows:

$$
\operatorname{vec}(\alpha)=\left(\begin{array}{c}
\operatorname{vec}(\mathbf{A}) \\
\operatorname{vec}(\mathbf{B})
\end{array}\right), \operatorname{vec}\left(\alpha^{\prime}\right)=\left(\begin{array}{c}
\operatorname{vec}\left(\mathbf{A}^{\prime}\right) \\
\operatorname{vec}\left(\mathbf{B}^{\prime}\right)
\end{array}\right), \operatorname{vec}(\xi)=\left(\begin{array}{c}
\operatorname{vec}(\mathbf{U}) \\
\operatorname{vec}(\mathbf{V}) \\
\operatorname{vec}(\mathbf{W})
\end{array}\right) .
$$

Then (7.1) for the mapping $d f_{\alpha}$ defined in (2.12) can be written in the form

$$
\mathbf{G}_{\alpha} \operatorname{vec}(\xi)=\operatorname{vec}\left(\alpha^{\prime}\right), \quad \operatorname{vec}(\xi) \in\left(\operatorname{null} \mathbf{G}_{\alpha}\right)^{\perp},
$$

where $\mathbf{G}_{\alpha}$ is an $\left(n^{2}+n m\right) \times\left(n^{2}+m^{2}+n m\right)$ real matrix of the form

$$
\mathbf{G}_{\alpha}=\left(\begin{array}{ccc}
\mathbf{I}_{n} \otimes \mathbf{A}-\mathbf{A}^{T} \otimes \mathbf{I}_{n} & 0 & \mathbf{I}_{n} \otimes \mathbf{B} \\
-\mathbf{B}^{T} \otimes \mathbf{I}_{n} & \mathbf{I}_{m} \otimes \mathbf{B} & 0
\end{array}\right),
$$

where $\mathbf{I}_{n} \otimes \mathbf{A}$ denotes the Kronecker product of matrices. The vector $\operatorname{vec}(\xi)$ satisfying the condition $\operatorname{vec}(\xi) \in\left(\text { null } \mathbf{G}_{\alpha}\right)^{\perp}$ can be expressed as follows:

$$
\operatorname{vec}(\xi)=\mathbf{G}_{\alpha}^{T} \mathbf{y},
$$


where $\mathbf{y}$ is a column vector of dimension $n^{2}+n m$. Substituting (7.5) into (7.3), we find

$$
\mathbf{G}_{\alpha} \mathbf{G}_{\alpha}^{T} \mathbf{y}=\operatorname{vec}\left(\alpha^{\prime}\right)
$$

The linear mapping $d f_{\alpha}$ considered in this paper has a nontrivial null-space. Hence, the matrix $\mathbf{G}_{\alpha} \mathbf{G}_{\alpha}^{T}$ is singular. Recall that $\left\{\alpha_{1}^{n}, \ldots, \alpha_{\ell}^{n}\right\}$ is a basis of Ker $d f_{\alpha}^{*}$. Then $\left\{\operatorname{vec}\left(\alpha_{1}^{n}\right), \ldots, \operatorname{vec}\left(\alpha_{\ell}^{n}\right)\right\}$ is a basis of the null-space of the symmetric matrix $\mathbf{G}_{\alpha} \mathbf{G}_{\alpha}^{T}$. Using the method described in [15], we construct the equation

$$
\left(\mathbf{G}_{\alpha} \mathbf{G}_{\alpha}^{T}+\sum_{i=1}^{\ell} \operatorname{vec}\left(\alpha_{i}^{n}\right)\left(\operatorname{vec}\left(\alpha_{i}^{n}\right)\right)^{T}\right) \mathbf{y}=\operatorname{vec}\left(\alpha^{\prime}\right),
$$

where the matrix in the left-hand side is nonsingular. Solution $\mathbf{y}$ of (7.7) can be found numerically using the standard codes. The obtained vector $\mathbf{y}$ is a particular solution of (7.6), which determines the unique solution (7.5) of (7.1).

For a matrix pair $\alpha=\alpha_{b}$, where $\alpha_{b}$ is the Brunovsky canonical form (5.5) considered in section 5 , we have $\ell=4$, and the pairs $\alpha_{1}^{n} q_{1}+\cdots+\alpha_{4}^{n} q_{4}$ are given by expressions (4.16), (4.21), (4.22), where $r=1, k_{1}=n-2, \mathbf{X}_{11}^{n}=0, \mathbf{Y}_{11}^{n}=0$, and $\mathbf{Y}_{12}^{n}=0$.

\section{REFERENCES}

[1] V. I. Arnold, Geometrical Methods in the Theory of Ordinary Differential Equations, Springer-Verlag, New York, 1983.

[2] D. L. Boley And W.-S. Lu, Measuring how far a controllable system is from an uncontrollable one, IEEE Trans. Automat. Control, AC-31 (1986), pp. 249-251.

[3] C.-T. Chen, Linear System Theory and Design, Holt, Rinehart, and Winston, New York, 1984.

[4] J. Demmel And B. KÅGström, The generalized Schur decomposition of an arbitrary pencil A$\lambda B$ : Robust software with error bounds and applications. I, ACM Trans. Math. Software, 19 (1993), pp. 160-174.

[5] J. Demmel and B. KÅgström, The generalized Schur decomposition of an arbitrary pencil A$\lambda B$ : Robust software with error bounds and applications. II, ACM Trans. Math. Software, 19 (1993), pp. 175-201.

[6] J. Ferrer, M. I. García-Planas, and F. Puerta, Brunowsky local form of a holomorphic family of pairs of matrices, Linear Algebra Appl., 253 (1997), pp. 175-198.

[7] F. R. Gantmacher, The Theory of Matrices, Vol. 1, Chelsea, New York, 1959.

[8] M. I. García-Planas and A. A. Mailybaev, Reduction to versal deformations of matrix pencils and matrix pairs with application to control theory, SIAM J. Matrix Anal. Appl., 24 (2003), pp. 943-962.

[9] I. Gohberg, P. Lancaster, and L. Rodman, Invariant Subspaces of Matrices with Applications, Wiley, New York, 1986.

[10] M. Gu, New methods for estimating the distance to uncontrollability, SIAM J. Matrix Anal. Appl., 21 (2000), pp. 989-1003.

[11] A. J. LAub, Survey of computational methods in control theory, in Electric Power Problems: The Mathematical Challenge, A. M. Erisman, K. W. Neves, and M. H. Dwarakanath, eds., SIAM, Philadelphia, 1980, pp. 231-260.

[12] R. V. Patel, A. J. Laub, and P. M. Van Doonen, Numerical Linear Algebra Techniques for Systems and Control, IEEE Press, New York, 1994.

[13] E. D. Sontag, Mathematical Control Theory: Deterministic Finite Dimensional Systems, Texts Appl. Math. 6, Springer-Verlag, New York, 1990.

[14] A. Tannenbaum, Invariance and System Theory: Algebraic and Geometric Aspects, Lecture Notes in Math. 845, Springer-Verlag, New York, 1981.

[15] V. A. Yakubovich and V. M. Starzhinskit, Parametric Resonance in Linear Systems, Nauka, Moscow, 1987 (in Russian). 